\title{
Association of the Aquaporin 3 Gene Polymorphism (rs2231231) with Epstein-Barr Virus-Associated Cancers in China
}

\author{
Jiayi Wang ${ }^{\mathrm{a}}$ Wen Liu $^{\mathrm{a}}$ Zhenzhen Zhao ${ }^{\mathrm{a}}$ Yan Zhang ${ }^{\mathrm{a}, \mathrm{b}}$ Bing Luo ${ }^{\mathrm{a}}$ \\ a'Department of Pathogenic Biology, Qingdao University Medical College, Qingdao, China; \\ ${ }^{b}$ Department of Clinical Laboratory, Central Hospital of Zibo, Zibo, China
}

\section{Keywords}

Aquaporin 3 - Epstein-Barr virus · Single-nucleotide polymorphism · Nasopharyngeal carcinoma - Lymphoma

\begin{abstract}
Background/Aims: Aquaporins are widely expressed in a variety of cancers and were found to play an important role in cell migration, angiogenesis, tumor formation, and tumor development. Thus, they have been proposed as a potential biomarker for some cancers. Accordingly, we analyzed the association between a single-nucleotide polymorphism locus of aquaporin 3 (rs2231231) and Epstein-Barr virus (EBV)associated nasopharyngeal carcinoma (EBVaNPC), lymphoma (EBVaL), and gastric carcinoma in China. Methods: Sequenom MassARRAY technique and polymerase chain reaction sequencing were used to test the genotype of 393 cases of patients with cancer and 148 normal control (NC) samples. A $X^{2}$ test or correction for continuity or the likelihood ratio was used for statistical analysis, and two-sided $p$ values $<0.05$ were considered a statistically significant difference. Results: We found that the genotype distributions were significantly different between either the EBVaNPC group and the NC group or the EBVaL case group and the
\end{abstract}

\section{KARGER}

(C) 2018 S. Karger AG, Basel

E-Mail karger@karger.com

www.karger.com/int
NC group. However, no statistical associations between rs2231231 and gastric carcinoma was observed in the current study. Conclusions: The AQP3 gene polymorphism is associated with a susceptibility to EBVaNPC and EBVaL, and the homozygous AA genotype is more frequently observed in individuals who develop EBVaNPC and EBVaL.

(c) 2018 S. Karger AG, Basel

\section{Introduction}

Epstein-Barr virus (EBV), also called human herpes virus 4 , which was discovered more than 40 years ago from a Burkitt's lymphoma biopsy, was the first virus to be known to have a direct association with human cancer [1]. EBV has 2 evident life cycles in the human host, one that produces new infectious virions and is called the lytic form of infection, and the other form that allows the virus to stay in a resting state for the lifetime of the host, called the latent form of infection [1]. EBV has a high exposure rate, with more than $95 \%$ of the populations worldwide being infected with EBV [2]. It is well known that EBV causes infectious mononucleosis, a frequent and relatively modest chronic inflammatory syndrome, 
Table 1. Clinical characteristics of the patients and healthy controls

\begin{tabular}{|c|c|c|c|c|c|c|c|}
\hline Characteristics & $\begin{array}{l}\text { NC } \\
(n=148)\end{array}$ & $\begin{array}{l}\text { EBVaNPC } \\
(n=90)\end{array}$ & $\begin{array}{l}\text { EBVnNPC } \\
(n=11)\end{array}$ & $\begin{array}{l}\text { EBVaGC } \\
(n=59)\end{array}$ & $\begin{array}{l}\text { EBVnGC } \\
(n=99)\end{array}$ & $\begin{array}{l}\text { EBVaL } \\
(n=89)\end{array}$ & $\begin{array}{l}\text { EBVnL } \\
(n=45)\end{array}$ \\
\hline Age, years & $55.84 \pm 12.42$ & $48.00 \pm 13.61$ & $52.22 \pm 15.51$ & $56.39 \pm 11.33$ & $59.24 \pm 11.78$ & $48.59 \pm 21.23$ & $48.98 \pm 17.6 \mathrm{~S}$ \\
\hline Male/female & $81 / 67$ & $50 / 40$ & $5 / 6$ & $37 / 22$ & $53 / 46$ & $48 / 41$ & $21 / 24$ \\
\hline $\begin{array}{l}\text { Types or grades } \\
\text { of the tumor }\end{array}$ & & $\begin{array}{l}\text { Type I: } 16 \\
\text { Type II: } 74\end{array}$ & $\begin{array}{l}\text { Type I: } 3 \\
\text { Type II: } 8\end{array}$ & $\begin{array}{l}\text { Well differentiated: } 0 \\
\text { Moderately differentiated: } 8 \\
\text { Poorly differentiated: } 51 \\
\text { Undifferentiated: } 0\end{array}$ & $\begin{array}{l}\text { Well differentiated: } 1 \\
\text { Moderately differentiated: } 22 \\
\text { Poorly differentiated: } 76 \\
\text { Undifferentiated: } 0\end{array}$ & $\begin{array}{l}\text { HL: } 23 \\
\text { NHL: } 66\end{array}$ & $\begin{array}{l}\text { HL: } 15 \\
\text { NHL: } 30\end{array}$ \\
\hline
\end{tabular}

The types and grades of the tumors are the common classification for nasopharyngeal carcinoma, gastric carcinoma, and lymphoma. NC, normal control; EBVaNPC, EBV-associated nasopharyngeal carcinoma; EBVnNPC, EBV-negative nasopharyngeal carcinoma; EBVaGC, EBV-associated gastric carcinoma; EBVnGC, EBV-negative gastric carcinoma; EBVaL, EBV-associated lymphoma; EBVnL, EBV-negative lymphoma; type I, keratinizing squamous cell carcinoma; type II, nonkeratinizing squamous cell carcinoma; HL, Hodgkin lymphoma; NHL, non-Hodgkin lymphoma.

and it is also related to other diseases and numerous cancers, including Burkitt lymphoma, Hodgkin lymphoma, nasopharyngeal carcinoma (NPC), and gastric carcinoma (GC) [3].

The aquaporins (AQPs) are a family of small $(\sim 30$ $\mathrm{kDa} /$ monomer) membrane transport proteins acting as a permeable tetramer located on a hydrophobic cell membrane $[4,5]$. Currently, at least 13 isoforms of AQPs (AQP $0-12$ ) have been identified in mammals and are considered responsible for transporting water and other molecules such as glycerol and urea [6]. Otherwise, the aquaporins mediate transmembrane signaling by conducting signaling molecules or coupling with other molecules as membrane proteins [7]. Many studies have also suggested that certain AQP subtypes might have an important effect on cell migration, carcinogenesis, and tumor progression and invasion; thus, they can be regarded as targets for a novel antitumor therapy $[8,9]$.

Aquaporin $3(A Q P 3)$ is a well-known aquaporin subtype that is wildly distributed in the esophagus, stomach, intestines, cervix, skin, head, and neck. Meanwhile, AQP3 has been found to be associated with numerous cancers [10]. Furthermore, studies suggested that $A Q P 3$ regulated the growth and spread of tumor cells through a number of signaling pathways. It seemed that in human GC induced by hyperglycemia, both the ERK and PI3K/AKT signaling pathways were involved in the upregulation of $A Q P 3 \mathrm{ex}-$ pression [11]. Another study pointed out that the AQP3 expression regulated by c-Met might affect the metastasis and invasion of human GC through the ERK signaling pathway [12]. Moreover, a recent study revealed that $A Q P 3$ had been proposed as a potential biomarker for GC [13].

Single-nucleotide polymorphisms (SNPs) have a great effect on human genome research. SNPs are usually used for the linkage analysis of diseases and can be used as genetic markers of pathogenic genes to locate unknown pathogenic genes. In addition, the SNP maps between susceptible populations and nonsusceptible populations are compared within the whole genome, and susceptibility genes are searched through an association analysis and linkage disequilibrium analysis. However, the results of the studies about $A Q P 3$ SNPs associated with tumors are rarely reported meaningful. In our study, we found that the associations of a $A Q P 3$ SNP locus with nasopharyngeal carcinoma or lymphoma, one possible reason for the gene expression or its alteration, might affect the diagnosis and prognosis of tumors.

\section{Materials and Methods}

\section{Specimens}

This study was performed in the Chinese population. Paraffinembedded and fresh tumor tissues were collected from the Department of Pathology of the Affiliated Hospital of Qingdao University and Qingdao Municipal Hospital. The specimens consisted of 101 cases of nasopharyngeal carcinoma (NPC) including $90 \mathrm{EBV}$ associated NPC (EBVaNPC) and $11 \mathrm{EBV}$-negative NPC (EBVnNPC); 134 cases of lymphoma including $89 \mathrm{EBV}$-associated lymphoma (EBVaL) and $45 \mathrm{EBV}$-negative lymphoma (EBVnL); and 158 cases of GC including 59 EBV-associated GC (EBVaGC) and 99 EBV-negative GC (EBVnGC). The clinical characteristics of the patients and healthy controls were listed in Table 1 . There were no statistical differences in age and gender between the healthy individuals and cancer patients, and between the types or grades of the tumors, there appeared to be no difference in EBV-associated cancers and EBV-negative cancers, neither in NPC, lymphoma, nor GC. None of the patients received hormone therapy, chemotherapy, or radiotherapy before surgery. EBV-positive specimens were identified by in situ hybridization for EBV-encoded small RNA 1, as described previously [14]. At least 2 independent pathologists determined the histological diagnosis and pathological stages of all 
Table 2. The distribution of the genotypes of AQP3 in NC, EBVaNPC, EBVnNPC, NPC, EBVaGC, EBVnGC, GC, EBVaL, EBVnL, and lymphoma

\begin{tabular}{|c|c|c|c|c|c|c|c|c|c|c|}
\hline $\begin{array}{l}\text { Genotypic } \\
\text { frequencies }\end{array}$ & $\begin{array}{l}\text { NC } \\
(n=148)\end{array}$ & $\begin{array}{l}\text { EBVaNPC } \\
(n=90)\end{array}$ & $\begin{array}{l}\text { EBVnNPC } \\
(n=11)\end{array}$ & $\begin{array}{l}\text { NPC } \\
(n=101)\end{array}$ & $\begin{array}{l}\text { EBVaGC } \\
(n=59)\end{array}$ & $\begin{array}{l}\text { EBVnGC } \\
(n=99)\end{array}$ & $\begin{array}{l}\text { GC } \\
(n=158)\end{array}$ & $\begin{array}{l}\text { EBVaL } \\
(n=89)\end{array}$ & $\begin{array}{l}\text { EBVnL } \\
(n=45)\end{array}$ & $\begin{array}{l}\text { Lymphoma } \\
(n=134)\end{array}$ \\
\hline AA & $7(4.73)$ & $10(11.11)$ & 0 & $10(9.9)$ & $4(6.78)$ & 9 (9.09) & $13(8.23)$ & $12(13.48)$ & 7 (15.56) & $19(14.18)$ \\
\hline $\mathrm{CC}$ & $77(52.03)$ & $55(61.11)$ & $9(81.82)$ & $64(63.37)$ & $28(47.46)$ & $45(45.45)$ & $73(46.20)$ & $48(53.93)$ & $23(51.11)$ & $71(52.99)$ \\
\hline$p$ & & 0.022 & & 0.017 & 0.758 & 0.313 & 0.358 & 0.031 & 0.062 & 0.012 \\
\hline
\end{tabular}

Values are presented as $n(\%)$. All $p$ values were compared with the NC group. NC, normal control; NPC, nasopharyngeal carcinoma; EBVaNPC, EBVassociated nasopharyngeal; EBVnNPC, EBV-negative nasopharyngeal; GC, gastric carcinoma; EBVaGC, EBV-associated gastric carcinoma; EBVnGC, EBVnegative gastric carcinoma; EBVaL, EBV-associated lymphoma; EBVnL, EBV-negative lymphoma.

Table 3. The distribution of the genotype and allele of $A Q P 3$ in EBVaNPC, NPC, and NC

\begin{tabular}{|c|c|c|c|}
\hline$A Q P 3($ rs 2231231) & EBVaNPC $(n=90)$ & $\mathrm{NC}(n=148)$ & NPC $(n=101)$ \\
\hline \multicolumn{4}{|l|}{ Genotype } \\
\hline AA & $10(11.11)$ & $7(4.73)$ & $10(9.90)$ \\
\hline $\mathrm{AC}$ & $25(27.78)$ & $64(43.24)$ & $27(26.73)$ \\
\hline OR [95\% CI] & $3.657[1.253-10.670]$ & & $3.386[1.167-9.828]$ \\
\hline$p$ & 0.014 & & 0.020 \\
\hline $\mathrm{CC}^{p}$ & $55(61.11)$ & $77(52.03)$ & $64(63.37)$ \\
\hline \multirow{2}{*}{$\begin{array}{l}\text { OR }[95 \% \mathrm{CI}] \\
p\end{array}$} & $2.000[0.717-5.580]$ & & $1.719[0.619-4.772]$ \\
\hline & 0.179 & & 0.294 \\
\hline \multicolumn{4}{|c|}{ Dominant model of $A$} \\
\hline $\mathrm{AA}+\mathrm{AC}$ & $35(38.89)$ & $71(47.97)$ & $37(36.63)$ \\
\hline $\mathrm{CC}$ & $55(61.11)$ & $77(52.03)$ & $64(63.37)$ \\
\hline$\underset{p}{\mathrm{OR}}[95 \% \mathrm{CI}]$ & $0.690[0.405-1.175]$ & & $0.627[0.374-1.052]$ \\
\hline$p$ & 0.172 & & 0.076 \\
\hline \multicolumn{4}{|l|}{ Recessive model of $A$} \\
\hline AA & $10(11.11)$ & $7(4.73)$ & $10(9.90)$ \\
\hline $\mathrm{AC}+\mathrm{CC}$ & $80(88.89)$ & $141(95.27)$ & $91(90.10)$ \\
\hline \multirow{2}{*}{$\begin{array}{l}\text { OR }[95 \% \mathrm{CI}] \\
p\end{array}$} & $2.518[0.922-6.872]$ & & $2.214[0.813-6.024]$ \\
\hline & 0.064 & & 0.112 \\
\hline \multicolumn{4}{|l|}{ Allele } \\
\hline \multirow{2}{*}{$\begin{array}{l}\text { A } \\
\text { C }\end{array}$} & $45(25)$ & $78(26.35)$ & $47(23.27)$ \\
\hline & $135(75)$ & $218(73.65)$ & $155(76.73)$ \\
\hline \multirow{2}{*}{$\underset{p}{\mathrm{OR}}[95 \% \mathrm{CI}]$} & $0.932[0.609-1.425]$ & & $0.847[0.559-1.285]$ \\
\hline & 0.744 & & 0.436 \\
\hline
\end{tabular}

collected tissues. The normal control (NC) group specimens were collected from peripheral blood samples of 148 healthy individuals, and this group did not receive any medications. This study was approved by the Medical Ethical Committee of Medical College of Qingdao University. All subjects gave informed consent, and all data were treated anonymously.

\section{DNA Extraction}

The DNA of paraffin-embedded tumor tissue was extracted and purified using a QIAamp DNA FFPE tissue kit (QIAGEN $\mathrm{GmbH}$, Hilden, Germany). A standard measure of proteinase K digestion and phenol-chloroform purification was used to extract the DNA of fresh tumor tissue and peripheral blood. 
Table 4. The distribution of the genotype and allele of $A Q P 3$ in EBVaL, EBVnL, lymphoma, and NC

\begin{tabular}{|c|c|c|c|c|}
\hline$A Q P 3$ (rs 2231231) & $\mathrm{NC}(n=148)$ & EBVaL $(n=89)$ & EBVnL $(n=45)$ & Lymphoma $(n=134)$ \\
\hline \multicolumn{5}{|l|}{ Genotype } \\
\hline $\mathrm{AA}$ & $7(4.73)$ & $12(13.48)$ & $7(15.56)$ & $19(14.18)$ \\
\hline $\mathrm{AC}$ & $64(43.24)$ & $29(32.58)$ & $15(33.33)$ & $44(32.84)$ \\
\hline OR $[95 \% \mathrm{CI}]$ & & $3.783[1.350-10.600]$ & $4.267[1.299-14.011]$ & $3.948[1.530-10.186]$ \\
\hline$p$ & & 0.008 & 0.030 & 0.003 \\
\hline $\mathrm{CC}$ & $77(52.03)$ & $48(53.93)$ & $23(51.11)$ & $71(52.99)$ \\
\hline OR $[95 \% \mathrm{CI}]$ & & $2.750[1.012-7.471]$ & $3.348[1.064-10.536]$ & $2.944[1.168-7.421]$ \\
\hline$p$ & & 0.041 & 0.068 & 0.018 \\
\hline \multicolumn{5}{|l|}{ Dominant model of $A$} \\
\hline $\mathrm{AA}+\mathrm{AC}$ & $71(47.97)$ & $41(46.07)$ & $22(48.89)$ & $63(47.01)$ \\
\hline $\mathrm{CC}$ & $77(52.03)$ & $48(53.93)$ & $23(51.11)$ & $71(52.99)$ \\
\hline OR $[95 \% \mathrm{CI}]$ & & $0.926[0.547-1.569]$ & $1.037(0.532-2.022)$ & $0.962(0.603-1.537)$ \\
\hline$p$ & & 0.776 & 0.914 & 0.872 \\
\hline \multicolumn{5}{|l|}{ Recessive model of $A$} \\
\hline AA & $7(4.73)$ & $12(13.48)$ & $7(15.56)$ & $19(14.18)$ \\
\hline $\mathrm{AC}+\mathrm{CC}$ & $141(95.27)$ & $77(86.52)$ & $38(84.44)$ & $115(85.82)$ \\
\hline OR $[95 \% \mathrm{CI}]$ & & $3.139[1.187-8.303]$ & $3.711(1.226-11.228)$ & $3.328(1.352-8.193)$ \\
\hline$p$ & & 0.016 & 0.034 & 0.006 \\
\hline \multicolumn{5}{|l|}{ Allele } \\
\hline A & $78(26.35)$ & $53(29.78)$ & $29(32.22)$ & $82(30.60)$ \\
\hline $\mathrm{C}$ & $218(73.65)$ & $125(70.22)$ & $61(67.78)$ & $186(69.40)$ \\
\hline OR $[95 \% \mathrm{CI}]$ & & $1.185[0.785-1.790]$ & $1.329(0.796-2.217)$ & $1.232(0.854-1.778)$ \\
\hline$p$ & & 0.420 & 0.276 & 0.264 \\
\hline
\end{tabular}

Values are presented as $n(\%)$, unless otherwise stated. All $p$ values were compared with the NC group. NC, normal control; EBVaL, EBV-associated lymphoma; EBVnL, EBV-negative lymphoma; OR, odds ratio; CI, confidence interval.

\section{Gene Polymorphism Identification}

Gene polymorphism was detected by Sequenom MassARRAY technique and polymerase chain reaction (PCR) sequencing. The products from SpectroCHIP were analyzed by MALDI-TOF mass spectrometer. Genotyping data were processed by Sequenom Launches TYPER 4.0 software. The genotyping was conducted at the Beijing Genomics Institute. The primers and probes used in the Sequenom MassARRAY technique are as follows: first PCR primer 5'-ACGTTGGATGACACCTGAGAGGGAAGTCTG-3', second PCRP 5'-ACGTTGGATGGCGAAGTGCCAGATTGCATC-3', unextension primer sequence $5^{\prime}$-ATCTGGTGACAGATTAGAC- $3^{\prime}$. The PCR reactions were performed with $2 \mu \mathrm{L}$ of DNA extract $(200 \mathrm{ng} / \mu \mathrm{L})$ in a $30-\mu \mathrm{L}$ reaction mixture containing $1 \times$ PCR reaction buffer, $0.4 \mu \mathrm{M}$ of each primer, $200 \mu \mathrm{M}$ dNTPs, and 1 U Pfu DNA polymerase (Takara Bio Inc., Kyoto, Japan). Cycle conditions were predenaturation at $95^{\circ} \mathrm{C}$ for $5 \mathrm{~min} ; 38$ cycles of $95^{\circ} \mathrm{C}$ for $30 \mathrm{~s}, 58^{\circ} \mathrm{C}$ for $30 \mathrm{~s}$, and $72^{\circ} \mathrm{C}$ for $1 \mathrm{~min}$; and final elongation at $72^{\circ} \mathrm{C}$ for $10 \mathrm{~min}$. The primers are as follows: $A Q P 3$ F 5'-AAAAGCTGGTTGTCGGCGAA-3', AQP3-R 5'-AATAACAGAGTCTCAGGCCCTC- $3^{\prime}$. The sequencing was conducted at the Beijing Genomics Institute.

\section{Statistics}

The genotype distributions of the control group were tested for accordance with the Hardy-Weinberg equilibrium. The $\chi^{2}$ test or correction for continuity or likelihood ratio were used for statistical analysis. Unconditional logistic regression was used to assess the odds ratio (OR) and $p$ values to identify the association between gene polymorphism and the risk of 3 types of carcinoma. $p$ values $<0.05$ were considered statistically significant. All statistical analyses were conducted using SPSS 17.0 (SPSS, Chicago, IL, USA).

\section{Results}

The genotype distributions of $A Q P 3$ rs2231231 in NPC (including EBVaNPC and EBVnNPC), GC (including EBVaGC and EBVnGC), lymphoma (including EB$\mathrm{VaL}$ and EBVnL), and NCs are shown in Table 2. The genotype distributions of the $\mathrm{NC}$ group have been verified to be consistent with the Hardy-Weinberg equilib$\operatorname{rium}\left(\chi^{2}=1.124, p>0.05\right)$. 
Table 5. The distribution of the genotype and allele of $A Q P 3$ in EBVaGC, EBVnGC, GC, and NC

\begin{tabular}{|c|c|c|c|c|}
\hline$A Q P 3$ (rs 2231231) & $\mathrm{NC}(n=148)$ & EBVaGC $(n=59)$ & EBVnGC $(n=99)$ & $\mathrm{GC}(n=158)$ \\
\hline \multicolumn{5}{|l|}{ Genotype } \\
\hline $\mathrm{AA}$ & $7(4.73)$ & $4(6.78)$ & $9(9.09)$ & $13(8.23)$ \\
\hline $\mathrm{AC}$ & $64(43.24)$ & $27(45.76)$ & $45(45.45)$ & $72(45.57)$ \\
\hline OR $[95 \% \mathrm{CI}]$ & & $1.354[0.366-5.011]$ & $1.829[0.634-5.271]$ & $1.651[0.620-4.392]$ \\
\hline$p$ & & 0.913 & 0.259 & 0.312 \\
\hline $\mathrm{CC}^{r}$ & $77(52.03)$ & $28(47.46)$ & $45(45.45)$ & $73(46.20)$ \\
\hline OR $[95 \% \mathrm{CI}]$ & & $1.571[0.427-5.780]$ & $2.200[0.767-6.312]$ & $1.959[0.740-5.183]$ \\
\hline$p$ & & 0.741 & 0.136 & 0.170 \\
\hline \multicolumn{5}{|l|}{ Dominant model of $A$} \\
\hline $\mathrm{AA}+\mathrm{AC}$ & $71(47.97)$ & $31(52.54)$ & $54(54.55)$ & $85(53.80)$ \\
\hline $\mathrm{CC}$ & $77(52.03)$ & $28(47.46)$ & $45(45.45)$ & $73(46.20)$ \\
\hline OR $[95 \% \mathrm{CI}]$ & & $1.201[0.656-2.197]$ & $1.301[0.781-2.168]$ & $1.263[0.806-1.979]$ \\
\hline$p$ & & 0.553 & 0.311 & 0.308 \\
\hline \multicolumn{5}{|l|}{ Recessive model of $A$} \\
\hline AA & $7(4.73)$ & $4(6.78)$ & $9(9.09)$ & $13(8.23)$ \\
\hline $\mathrm{AC}+\mathrm{CC}$ & $141(95.27)$ & $55(93.52)$ & $90(90.9)$ & $145(91.77)$ \\
\hline OR $[95 \% \mathrm{CI}]$ & & $1.465[0.412-5.203]$ & $2.014[0.725-5.600]$ & $1.806[0.700-4.659]$ \\
\hline$p$ & & 0.802 & 0.172 & 0.216 \\
\hline \multicolumn{5}{|l|}{ Allele } \\
\hline A & $78(26.35)$ & $35(29.66)$ & $63(31.81)$ & $98(31.01)$ \\
\hline $\mathrm{C}$ & $218(73.65)$ & $83(70.34)$ & $135(68.18)$ & $218(68.99)$ \\
\hline OR $[95 \% \mathrm{CI}]$ & & $1.179[0.735-1.890]$ & $1.304[0.878-1.937]$ & $1.256[0.884-1.786]$ \\
\hline$p$ & & 0.495 & 0.187 & 0.203 \\
\hline
\end{tabular}

Values are presented as $n(\%)$, unless otherwise stated. All $p$ values were compared with the NC group. NC, normal control; GC, gastric carcinoma; EBVaGC, EBV-associated gastric carcinoma; EBVnGC, EBV-negative gastric carcinoma; OR, odds ratio; $\mathrm{CI}$, confidence interval.

\section{Associations of rs2231231 with NPC}

Genotype distributions were significantly different between NPC and NC $\left(\chi^{2}=8.192, p=0.017\right)$, mainly between EBVaNPC and NC $\left(\chi^{2}=7.603, p=0.022\right)$. We merged EBVaNPC and EBVnNPC into the NPC group because the genotype distributions showed no difference between EBVaNPC and EBVnNPC $\left(\chi^{2}=3.296, p=0.192\right)$. As shown in Table 3, compared with NC, patients with EBVaNPC exhibited a higher frequency of the AA genotype $(p=0.014, \mathrm{OR}=3.657,95 \%$ confidence interval [CI] 1.253-10.670). Furthermore, in the super-dominant model, the distributions of the homozygous genotype (CC and $\mathrm{AA}$ ) and heterozygous genotype $(\mathrm{AC})$ were obviously different between NPC and NC $(p=0.008$, OR $=$ $2.088,95 \%$ CI 1.208-3.611).

\section{Associations of rs2231231 with Lymphoma}

The lymphoma group, especially the EBVaL group, exhibited different genotype distributions than the NC group (lymphoma vs. NC: $\chi^{2}=8.812, p=0.006$; EBVaL vs. NC: $\chi^{2}=6.959, p=0.031$ ). As was shown in Table 4 , in the additive model, a significant difference was found between lymphoma and NC ( $p=0.018, \mathrm{OR}=2.944,95 \%$ CI 1.168-7.421). The frequency of the AA genotype in the lymphoma group was higher than that in the NC group; however, there was no difference between the NC and EBVnL groups. In the recessive model of $\mathrm{A}$, we discovered visible differences between the $\mathrm{NC}$ group and the other 3 groups, while the frequency of the AA genotype in all case groups were higher than that in the NC group (lymphoma vs. NC: $p=0.006, \mathrm{OR}=3.328,95 \%$ CI $1.352-$ 8.193; EBVaL vs. NC: $p=0.016, \mathrm{OR}=3.139,95 \% \mathrm{CI}$ 1.187-8.303; EBVnL vs. NC: $p=0.034$, OR $=3.711,95 \%$ CI 1.226-11.228). However, we did not find any difference between EBVaL and EBVnL. 
Associations of rs 2231231 with GC

No statistical association between rs2231231 and GC was observed in the current study (Table 5). There was no significant difference in the distributions of the genotypes between the gastric cancer group (neither EBVaGC nor EBVnGC $\left.\left[\chi^{2}=0.279, p=0.870\right]\right)$ and the NC group $\left(\chi^{2}=\right.$ $2.053, p=0.358)$. There was no substantial difference in the allele frequency between the gastric cancer group and the NC group as well. These statistical analyses demonstrated that there was no evidence for associations between AQP3 (rs2231231) and the development of GC.

\section{Discussion}

Aquaporins (AQPs), a kind of small integral membrane water transport proteins, have important roles in cell proliferation, migration, and angiogenesis in various human cancers [15]. The mutation site of the SNP (rs2231231) is located in the chromosome 9 intron region; the ancestor allele is $\mathrm{C}$ while the minor allele is $\mathrm{A}$. Our study explored the relation between the AQP3 SNP, EBV infection, and cancers. In our present study, the AA genotype of rs2231231 showed a higher frequency in both EBVaNPC and EBVaL than in the NC group, whereas there was no difference between EBVaGC and the NC group, indicating that AA might be a risk factor for some specific EBV-associated tumors.

The AQP3 gene encodes aquaporin 3, which plays a crucial role in the formatting of the human hydration barrier and the regulation of water and osmolyte homeostasis in the human body, was found to be expressed in many human tissues [16]. In a study of $A Q P 3$ expression, they found that $A Q P 3$ expression had a good correlation with the survival of the patients with HER2-overexpressing early breast cancer. Meanwhile, they revealed that $A Q P 3$ expression could be an independent prognostic marker of disease-free survival. At the sametime, 7SNPs (rs10813981, rs34391490, rs2228332, rs2227285, rs591810, rs17553719, and rs3860987) of AQP3 were selected for further study; however, none of these SNPs was found to have associations with $A Q P 3$ variants and the expression of $A Q P 3$ in tumor tissues or survival [17]. A case-control study of polymorphisms in xenobiotic and arsenic metabolism genes and arsenic-related bladder cancer in New Hampshire suggested that AQP3 (rs2228332) homozygous carriers who exposed to higher arsenic levels might have an increased risk of bladder cancer [18].

One significant finding in our study was the fact that the genotype distributions in the nasopharyngeal carcinoma case group and EBV-associated nasopharyngeal carcinoma (EBVaNPC) case group was obviously different from the NC group. We merged EBVaNPC and EBVnNPC because there were no differences between them, and some studies revealed that most patients with nasopharyngeal carcinoma were suffering from EBVaNPC $[19,20]$. Thus, the difference between NPC and NC might be caused by the combined effect of both EBV and carcinoma. However, the genotype distributions presented no difference between the GC case group and the NC group. This phenomenon may be caused by the variety in the patterns of latent protein expression of EBV. EBVaGC has a unique latent protein expression type, while EBV could not express latent membrane protein 1 in most of the EBVaGC [1].

SNPs have a wide distribution and can be stably inherited; therefore, they are considered important biomarkers [17]. However, the application of individual SNPs is limited. In fact, single-nucleotide mutations on introns rarely have a substantial effect on gene expression but might influence the structure of a gene. Some studies speculated that SNPs might influence the miRNA transcription, processing, and/or the fidelity of the miRNA-mRNA interaction when the SNPs are located in the encoding miRNA sequence or the $3^{\prime} \mathrm{UTR}$ regions of the corresponding binding sites of genes [21].

In conclusion, our present results demonstrated the association between the AQP3 polymorphism (rs2231231) and nasopharyngeal carcinoma, lymphoma, and GC. Moreover, the AA genotype is associated with a susceptibility to EBVaNPC and lymphoma. In our future work, we will explore the relation between $A Q P 3$ SNPs and EBV, especially the regulatory mechanism of EBV towards the $A Q P 3$ gene.

\section{Acknowledgement}

This research was supported by the National Natural Science Foundation of China (NSFC 81571995) and the Natural Science Foundation of Shandong Province (ZR2017BH106).

\section{Disclosure Statement}

The authors have no conflicts of interest to disclose.

\section{Author Contributions}

B.L. conceived the project and designed the experiments. J.W., W.L., Y.Z., and Z.Z. participated in the performance of the experiments and the drafting of the manuscript. All authors read and approved the final manuscript. 


\section{References}

1 Pattle SB, Farrell PJ: The role of Epstein-Barr virus in cancer. Expert Opin Biol Ther 2006; 6:1193-1205.

2 Luzuriaga K, Sullivan JL: Infectious mononucleosis. N Engl J Med 2010;362:1993-2000.

3 Kim KY, Le QT, Yom SS, Ng RHW, Chan KCA, Bratman SV, Welch JJ, Divi RL, Petryshyn RA, Conley BA: Clinical utility of Epstein-Barr virus DNA testing in the treatment of nasopharyngeal carcinoma patients. Int J Radiat Oncol Biol Phys 2017;98:996-1001.

4 Carbrey JM, Agre P: Discovery of the aquaporins and development of the field. Handb Exp Pharmacol 2009;190:3-28.

5 Agre $\mathrm{P}$, King LS, Yasui M, Guggino WB, Ottersen OP, Fujiyoshi Y, Engel A, Nielsen S: Aquaporin water channels - from atomic structure to clinical medicine. J Physiol 2002; 542:3-16.

6 Zhu C, Chen Z, Jiang Z: Expression, distribution and role of aquaporin water channels in human and animal stomach and intestines. Int J Mol Sci 2016;17:E1399

7 Rojek AM, Skowronski MT, Füchtbauer EM, Füchtbauer AC, Fenton RA, Agre P, Frøkiaer J, Nielsen S: Defective glycerol metabolism in aquaporin 9 (AQP9) knockout mice. Proc Natl Acad U S A 2007;104:3609-3614.

8 Hoque MO, Soria JC, Woo J, Lee T, Lee J, Jang SJ, Upadhyay S, Trink B, Monitto C, Desmaze C, Mao L, Sidransky D, Moon C: Aquaporin 1 is overexpressed in lung cancer and stimu- lates NIH-3T3 cell proliferation and anchorage-independent growth. Am J Pathol 2006; 168:1345-1353.

9 Chae YK, Woo J, Kim MJ, Kang SK, Kim MS, Lee J, Lee SK, Gong G, Kim YH, Soria JC, Jang SJ, Sidransky D, Moon C: Expression of aquaporin 5 (AQP5) promotes tumor invasion in human non small cell lung cancer. PloS One 2008;3:e2162.

10 Wang G, Gao F, Zhang W, Chen J, Wang T, Zhang G, Shen L: Involvement of aquaporin 3 in Helicobacter pylori-related gastric diseases. PloS One 2012;7:e49104.

11 Zhou Y, Wang Y, Wang S, Shen L: Hyperglycemia promotes human gastric carcinoma progression via aquaporin 3. Dig Dis Sci 2015; 60:2338-2345.

12 Wang J, Gui Z, Deng L, Sun M, Guo R, Zhang W, Shen L: c-Met upregulates aquaporin 3 expression in human gastric carcinoma cells via the ERK signalling pathway. Cancer Lett 2012;319:109-117.

13 Zhao H, Yang X, Zhou Y, Zhang W, Wang Y, Wen J, Zhang Z, Shen L: Potential role of aquaporin 3 in gastric intestinal metaplasia. Oncotarget 2015;6:5370.

14 Tokunaga M, Land CE, Uemura Y, Tokudome T, Tanaka S, Sato E: Epstein-Barr virus in gastric carcinoma. Am J Pathol 1993;143: $1250-1254$.

15 Nagaraju GP, Basha R, Rajitha B, Alese OB, Alam A, Pattnaik S, El-Rayes B: Aquaporins: their role in gastrointestinal malignancies. Cancer Lett 2016;373:12-18.

16 Mobasheri A, Wray S, Marples D: Distribution of AQP2 and AQP3 water channels in human tissue microarrays. J Mol Histol 2005; 36:1-14.

17 Kang S, Chae YS, Lee SJ, Kang BW, Kim JG, Kim WW, Jung JH, Park HY, Jeong JH, Jeong JY, Park JY: Aquaporin 3 expression predicts survival in patients with HER2-positive early breast cancer. Anticancer Res 2015;35:27752782.

18 Lesseur C, Gilbert-Diamond D, Andrew AS, Ekstrom RM, Li Z, Kelsey KT, Marsit CJ, Karagas MR: A case-control study of polymorphisms in xenobiotic and arsenic metabolism genes and arsenic-related bladder cancer in New Hampshire. Toxicol Lett 2012;210: 100-106.

19 Tsao SW, Tsang CM, Lo KW: Epstein-Barr virus infection and nasopharyngeal carcinoma. Philos Trans R Soc B Biol Sci 2017;372: 1732.

20 Tsang CM, Tsao SW: The role of Epstein-Barr virus infection in the pathogenesis of nasopharyngeal carcinoma. Virol Sin 2015;30: 107-121.

21 Vodicka P, Pardini B, Vymetalkova V, Naccarati A: Polymorphisms in non-coding RNA genes and their target sites as risk factors of sporadic colorectal cancer. Adv Exp Med Biol 2016;937:123-149. 